\title{
Comparison of the diagnostic efficacy of white light endoscopy and magnifying endoscopy with narrow band imaging for early gastric cancer: a meta-analysis
}

\author{
Qiang Zhang ${ }^{1} \cdot$ Fei Wang ${ }^{1} \cdot$ Zhen-Yu Chen $^{1} \cdot$ Zhen Wang $^{1} \cdot$ Fa-Chao Zhi ${ }^{1}$. \\ Si-De Liu ${ }^{1} \cdot$ Yang Bai ${ }^{1}$
}

Received: 29 January 2015/Accepted: 14 April 2015/Published online: 29 April 2015

(C) The International Gastric Cancer Association and The Japanese Gastric Cancer Association 2015

\begin{abstract}
Objective Magnifying endoscopy with narrow band imaging (ME-NBI) is widely used in gastroscopy, especially in the diagnosis of early gastric cancer. The purpose of this meta-analysis is to compare the diagnostic efficacy of white light imaging (WLI) and that of ME-NBI for early gastric cancer.

Methods PubMed/MEDLINE, EMBASE, and the Cochrane Library were searched to identify studies which met the inclusion criteria. A random-effects model was used to calculate overall sensitivity, specificity, and the area under the receiver operating characteristic curve (AUC) to assess the diagnostic efficacy of WLI and MENBI in early gastric cancer. Sensitivity analysis was performed to assess the stability of the results.

Results Ten studies met the inclusion criteria, and included 1724 patients and 2153 lesions. The pooled sensitivity, specificity, and AUC for the diagnosis of early gastric cancer using WLI were 0.48 [95\% confidence interval (CI) $\left.0.39-0.57 ; \quad I^{2}=78.6 \%\right], \quad 0.67 \quad(95 \%$ CI $\left.0.62-0.71 ; \quad I^{2}=81.9 \%\right)$, and 0.62 , respectively. The pooled sensitivity, specificity, and AUC using ME-NBI were 0.83 (95\% CI $\left.0.79-0.87 ; I^{2}=79.8 \%\right), 0.96(95 \%$ CI $0.95-0.97 ; I^{2}=89.3 \%$ ), and 0.96 , respectively. The
\end{abstract}

Qiang Zhang and Fei Wang are contributed equally to this work.

Electronic supplementary material The online version of this article (doi:10.1007/s10120-015-0500-5) contains supplementary material, which is available to authorized users.

Qiang Zhang

4024313@qq.com

1 Department of Gastroenterology, Nanfang Hospital, Southen Medical University, Guangzhou, Guangdong, China studies showed a high degree of heterogeneity. Further sensitivity analysis was mainly performed for the studies of small lesions (mean size $10 \mathrm{~mm}$ or less) and the studies with a the score of 12 points or greater in the literature quality assessment, and the AUCs for ME-NBI for diagnosis of early gastric cancer were between 0.93 and 0.98 , which suggested that the diagnostic value was still high and stable.

Conclusion Compared with WLI, ME-NBI can effectively diagnose early gastric cancer.

Keywords Gastric cancer - Magnifying endoscopy · Narrow band imaging - White light endoscopy

\section{Introduction}

In 2012, the number of new cases of gastric cancer was nearly one million, accounting for $6.8 \%$ of new cancer cases, and gastric cancer has become the fifth commonest malignancy in the world, after lung cancer, breast cancer, colorectal cancer, and prostate cancer. Gastric cancer has become the third greatest cause of death from cancer, after lung cancer and liver cancer [1,2]. The Japanese Research Society of Gastric Cancer classifies gastric cancer into early and advanced stages on the basis of the depth of invasion of gastric lesions. Early gastric cancer is defined as a lesion confined to the mucosa or submucosa, regardless of lymph node metastasis [3]. An effective method to improve the prognosis of patients with gastric cancer is early detection and treatment [4-6]. After endoscopic resection of early gastric cancer which is considered as curative, the 5-year survival rate is more than $95 \%$ [4]. Some patients with early-stage gastric cancer who receive minimally invasive surgery by endoscopy have a better 
prognosis and less surgical trauma [7-9]. The 5-year survival rate in advanced gastric cancer patients is very low, even after surgery-predominant combined treatment, and the survival rate of gastric cancer patients in Western countries is only $20-30 \%[10,11]$. Therefore, early diagnosis and treatment is important to improve the 5-year survival rate and prognosis of gastric cancer.

Screening methods for gastric cancer include gastrointestinal tumor markers, X-ray barium radiography, and endoscopic examination. Endoscopic examination is still the most direct and effective method [12]. Traditional gastroscopy includes normal white light imaging (WLI), and indigo carmine dye endoscopy. With the development of endoscopic techniques, magnifying endoscopy with narrow band imaging (ME-NBI) has been used in the diagnosis of gastric diseases, especially early gastric cancer. Studies have reported the sensitivity of ME-NBI for diagnosis of early gastric cancer ranges from 0.60 to 0.97 [13, 14], which shows there are distinct differences among the studies. Therefore, the purpose of this study was to conduct a meta-analysis to compare the diagnostic efficacy of WLI and ME-NBI for early gastric cancer.

\section{Materials and methods}

\section{Search strategy}

PubMed/MEDLINE, EMBASE, and the Cochrane Library were searched for studies focusing on the diagnostic efficacy of ME-NBI and WLI for gastric cancer and highgrade intraepithelial neoplasia published in English until October 2014. The search keywords for the endoscopic technique were "narrow band imaging" or "NBI" and "white light" or "WLI." The search keywords for disease were "high grade neoplasia" or "high grade intraepithelial neoplasia," "gastric cancer" or "stomach cancer," and "stomach neoplasm" or "gastric neoplasm." The two sets of keywords were combined individually, and searched through Medical Subject Headings (MeSH) terms and free text. In addition, references in the included literature were searched manually to identify relevant studies. First, the title and abstract of identified articles were read to exclude studies which did not meet the inclusion criteria. Then, the full text of the remaining studies was carefully reviewed to decide whether to include the study (Fig. 1).

\section{Literature inclusion and exclusion criteria}

The inclusion criterias were as follows: (1) the goal of the study was to evaluate the diagnostic efficacy of ME-NBI and WLI for early gastric cancer, including high-grade intraepithelial neoplasia considered as early gastric cancer;

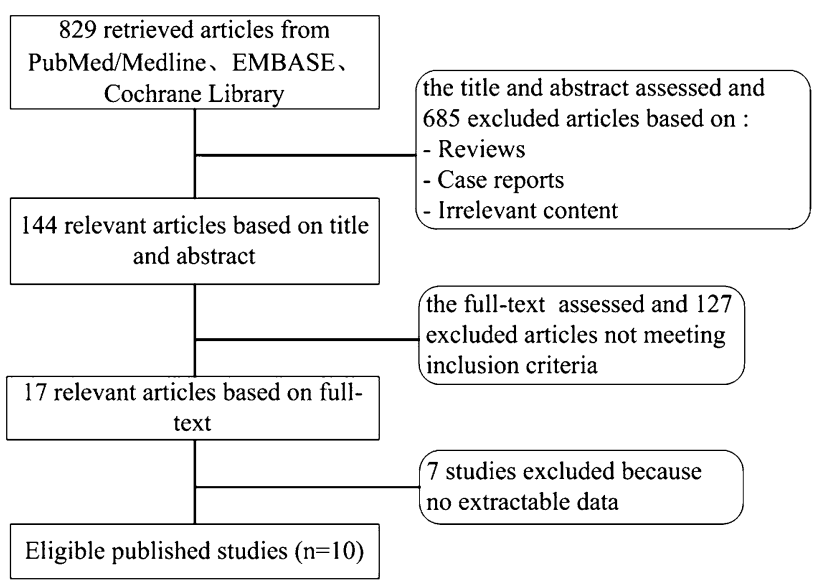

Fig. 1 Flowchart showing the process for selecting eligible studies

(2) the diagnostic gold standard was the pathological diagnosis of a biopsy, endoscopic submucosal dissection (ESD), or surgical specimen; (3) the number of true positive, true negative, false positive, and false negative gastric cancer diagnoses determined by ME-NBI and WLI could be obtained directly or indirectly.

The exclusion criterias were as follows: (1) the pathological result was not the gold standard for diagnosis; (2) the statistical methods were inappropriate; (3) the sample data were incomplete, and the number of true positive, true negative, false positive, and false negatives could not be obtained; (4) data on the observation parameters could not be combined; (5) case reports, review articles, meta-analyses, editorials, and articles for which only the abstract could be obtained.

\section{Data extraction}

A fourfold table was set up for the gastric cancer diagnosed by ME-NBI, WLI, and pathological data to record the number of true positive, false positive, true negative, and false negative cases in each study. Other data recorded included the authors, publication year, basic patient characteristics, the criteria of endoscopic diagnosis, and pathological diagnoses. Data extraction was performed by two independent investigators, and disagreement was resolved by discussion.

\section{Quality evaluation}

As shown in the electronic supplementary material ("supplemental material 1"), the quality and bias of studies were evaluated by Quality Assessment of Diagnostic Accuracy Studies (QUADAS) criteria [15]. Each assessment was evaluated as "yes," "no," or "unclear." The assessment was performed by two independent investigators, and disagreement was resolved by discussion. 


\section{Data analysis}

Meta-DiSc 1.4 was used to examine the heterogeneity of the data, and the combined effect size. $I^{2}$ was used to examine heterogeneity, with $I^{2}<25 \%$ indicating low heterogeneity, $25 \%<I^{2}<50 \%$ indicating moderate heterogeneity, and $I^{2}>50 \%$ indicating high heterogeneity among studies. In a diagnostic test, the threshold effect is an important cause of heterogeneity. The Spearman correlation coefficient of sensitivity and 1-specificity was calculated to estimate the threshold effect among the studies included. If there was no heterogeneity and threshold effect among the studies included, a fixed-effects model was used. If heterogeneity existed among studies, a random-effects model was used to combine the effect size.

If there was large heterogeneity among studies, a random-effects model was used to calculate the sensitivity, specificity, likelihood ratio, diagnostic odds ratio (DOR), and area under the receiver operating characteristic curve (AUC) from pooling of all the true positive, false positive, true negative, and false negative data.

Meta-regression analysis was performed to explore the sources of heterogeneity if significant heterogeneity was found. Next, the stability of the study results was assessed by sensitivity analysis. Publication bias was analyzed using the weighted linear regression model and the Deeks funnel plot provided by Stata 12. A two-tailed $P<0.05$ was considered statistically significant.

\section{Results}

\section{Literature selection and quality assessment}

The literature screening process is shown in Fig. 1. After reading the title and abstract, we identified 144 articles. Among them, the full texts of ten articles were eventually reviewed, which met the inclusion criterias. The ten articles evaluated the diagnostic value of ME-NBI in early gastric cancer, and four of them [16-19] also evaluated the diagnostic value of WLI in early gastric cancer. Except for the study of Fujiwara et al. [20], the other studies also investigated high-grade intraepithelial neoplasia. The studies of Tsuji et al. [23] and Maki et al. [17] investigated gastric adenoma and gastric adenocarcinoma. Six studies investigated small lesions with mean size of $10 \mathrm{~mm}$ or less [14, 16, 18-21]. The results of the quality assessment of each study are shown in Table 1. Detailed information regarding each study is shown in Table 2.

\section{The value of ME-NBI in the diagnosis of early gastric cancer}

The ten studies included 1724 patients and 2153 lesions. Each study assessed the diagnostic efficacy of ME-NBI for early gastric cancer lesions, and four studies [16-18, 23 ] also evaluated the diagnostic efficacy of WLI in patients with early gastric cancer. As shown in Fig. 2, there was large heterogeneity among the studies. The Spearman correlation coefficient obtained for the assessment of the threshold effect was 0.237 ( $P=0.510)$, which indicated there was no threshold effect among the studies. With use of a random-effects model, the sensitivity and specificity of each study regarding ME-NBI in the diagnosis of gastric cancer lesion were combined, and the pooled sensitivity was 0.83 [95 \% confidence interval (CI) $0.79-0.87 ; I^{2}=79.8 \%$, the pooled specificity was $0.96\left(95 \%\right.$ CI $\left.0.95-0.97 ; I^{2}=89.3 \%\right)$, the DOR was 88.83 (95\% CI 36.53-215.99; $I^{2}=75.8 \%$ ), the AUC was 0.96 (standard error 0.01 ), and $Q^{*}$ was 0.90 (standard error 0.02).

Next, on per-patient analysis we combined the diagnostic efficacy of ME-NBI in gastric cancer using a random-effects model ("supplemental material 2" in the
Table 1 Quality assessment by the Quality Assessment of Diagnostic Accuracy Studies (QUADAS) tool of the ten studies included in the metaanalysis

\begin{tabular}{|c|c|c|c|c|c|c|c|c|c|c|c|c|c|c|c|}
\hline \multirow[t]{2}{*}{ Study } & \multicolumn{14}{|c|}{ Question number } & \multirow[t]{2}{*}{ Score } \\
\hline & 1 & 2 & 3 & 4 & 5 & 6 & 7 & 8 & 9 & 10 & 11 & 12 & 13 & 14 & \\
\hline Li et al. [13] & $\mathrm{N}$ & $\mathrm{Y}$ & $\mathrm{Y}$ & Y & $\mathrm{Y}$ & $\mathrm{Y}$ & $\mathrm{Y}$ & $\mathrm{Y}$ & $\mathrm{Y}$ & $\mathrm{Y}$ & $\mathrm{Y}$ & $\mathrm{Y}$ & $\mathrm{Y}$ & $\mathrm{Y}$ & 13 \\
\hline Fujiwara et al. [20] & $\mathrm{N}$ & $\mathrm{N}$ & $\mathrm{Y}$ & $\mathrm{Y}$ & $\mathrm{Y}$ & $\mathrm{Y}$ & $\mathrm{Y}$ & $\mathrm{Y}$ & $\mathrm{Y}$ & $\mathrm{Y}$ & $\mathrm{Y}$ & $\mathrm{N}$ & $\mathrm{Y}$ & $\mathrm{Y}$ & 11 \\
\hline Yao et al. [14] & $\mathrm{Y}$ & $\mathrm{Y}$ & $\mathrm{Y}$ & Y & $\mathrm{U}$ & $\mathrm{Y}$ & $\mathrm{Y}$ & $\mathrm{Y}$ & $\mathrm{Y}$ & $\mathrm{Y}$ & $\mathrm{Y}$ & $\mathrm{Y}$ & $\mathrm{Y}$ & $\mathrm{Y}$ & 13 \\
\hline Tao et al. [21] & $\mathrm{N}$ & $\mathrm{Y}$ & $\mathrm{Y}$ & $\mathrm{Y}$ & $\mathrm{Y}$ & $\mathrm{Y}$ & $\mathrm{Y}$ & $\mathrm{Y}$ & $\mathrm{Y}$ & $\mathrm{Y}$ & $\mathrm{U}$ & $\mathrm{Y}$ & $\mathrm{Y}$ & $\mathrm{Y}$ & 12 \\
\hline Kato et al. [16] & $\mathrm{N}$ & $\mathrm{Y}$ & $\mathrm{Y}$ & $\mathrm{Y}$ & $\mathrm{Y}$ & $\mathrm{Y}$ & $\mathrm{Y}$ & $\mathrm{Y}$ & $\mathrm{Y}$ & $\mathrm{U}$ & $\mathrm{U}$ & $\mathrm{Y}$ & $\mathrm{Y}$ & $\mathrm{Y}$ & 11 \\
\hline Tsuji et al. [23] & $\mathrm{N}$ & $\mathrm{N}$ & $\mathrm{Y}$ & $\mathrm{Y}$ & $\mathrm{Y}$ & $\mathrm{Y}$ & $\mathrm{Y}$ & $\mathrm{Y}$ & $\mathrm{Y}$ & $\mathrm{Y}$ & $\mathrm{Y}$ & $\mathrm{Y}$ & $\mathrm{Y}$ & $\mathrm{Y}$ & 12 \\
\hline Maki et al. [17] & $\mathrm{N}$ & $\mathrm{N}$ & $\mathrm{Y}$ & $\mathrm{Y}$ & $\mathrm{Y}$ & $\mathrm{Y}$ & $\mathrm{Y}$ & $\mathrm{Y}$ & $\mathrm{Y}$ & $\mathrm{U}$ & $\mathrm{U}$ & $\mathrm{Y}$ & $\mathrm{Y}$ & $\mathrm{Y}$ & 10 \\
\hline Ezoe et al. [18] & $\mathrm{N}$ & $\mathrm{Y}$ & Y & $\mathrm{Y}$ & Y & Y & Y & $\mathrm{Y}$ & $\mathrm{Y}$ & $\mathrm{Y}$ & Y & $\mathrm{Y}$ & $\mathrm{Y}$ & $\mathrm{Y}$ & 13 \\
\hline Ezoe et al. [19] & $\mathrm{N}$ & $\mathrm{N}$ & Y & Y & Y & Y & Y & Y & $\mathrm{Y}$ & $\mathrm{U}$ & $\mathrm{U}$ & $\mathrm{Y}$ & $\mathrm{Y}$ & Y & 10 \\
\hline Liu et al. [22] & $\mathrm{Y}$ & $\mathrm{Y}$ & $\mathrm{Y}$ & $\mathrm{Y}$ & $\mathrm{Y}$ & $\mathrm{Y}$ & $\mathrm{Y}$ & $\mathrm{Y}$ & $\mathrm{Y}$ & $\mathrm{Y}$ & $\mathrm{Y}$ & $\mathrm{Y}$ & $\mathrm{Y}$ & $\mathrm{Y}$ & 14 \\
\hline
\end{tabular}

The responses to the 14 question items were "yes" $(Y)$, "no" $(N)$, or "unclear" $(U)$. 


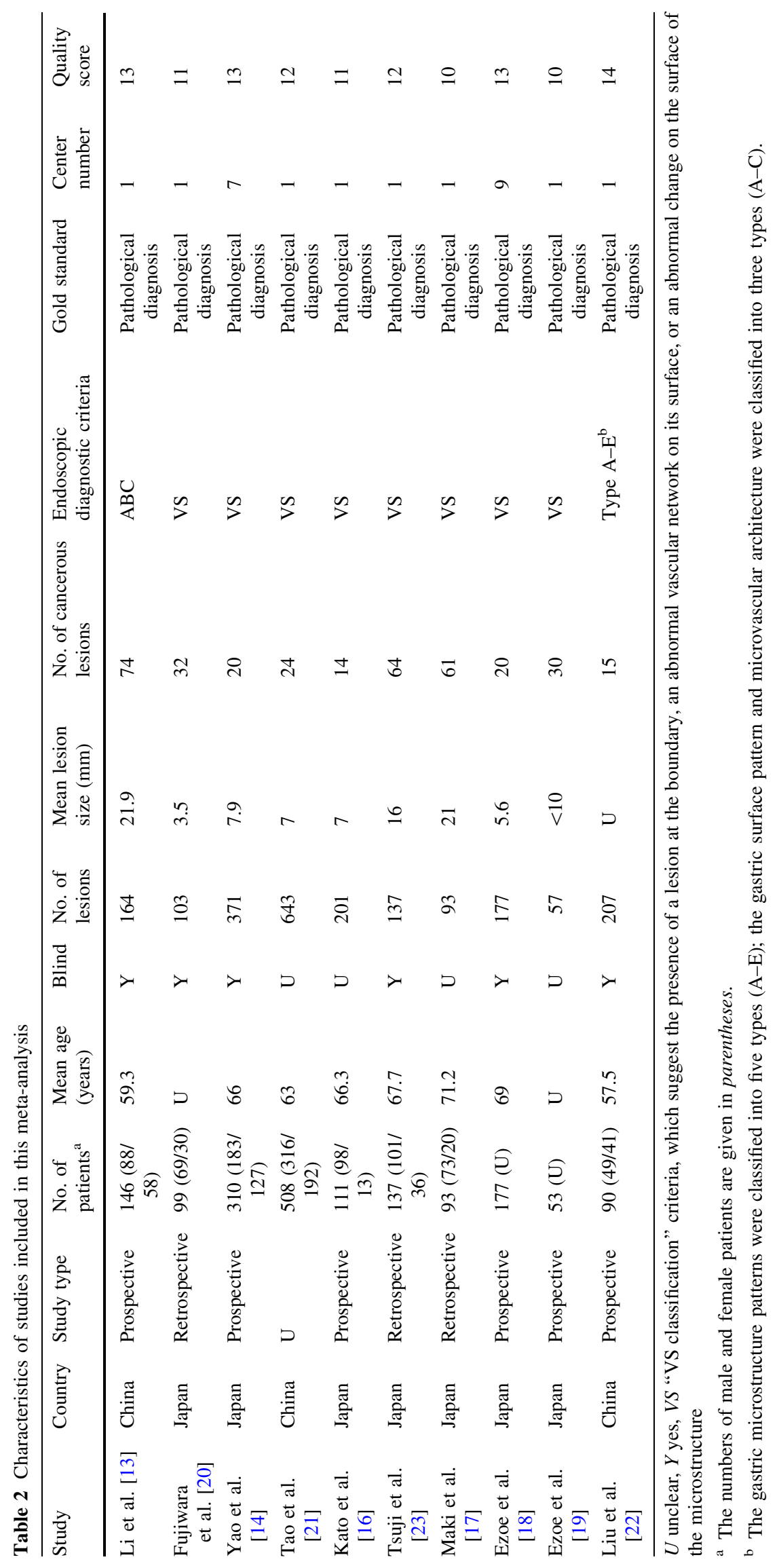




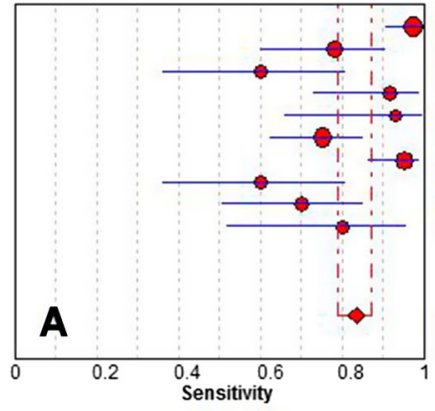

H-y. Li 2012 Shoko Fujiwara 2014 Kenshi Yao 2014 Guo Tao 2013 Masayuki Kato 2010 Yosuke Tsuji 2012 YASUMASAEZOE 2011 Yasumasa Ezoe 2010 Hong Liu 2014

Pooled Sensitivity $=0.83(0.79$ to 0.87$)$ Chi-square $=44.61 ;$ df $=9(p=0.0000)$ Chi-square $=44.61 ;$ df $=9(p=0.0000)$
Inconsistency (l-square $)=79.8 \%$
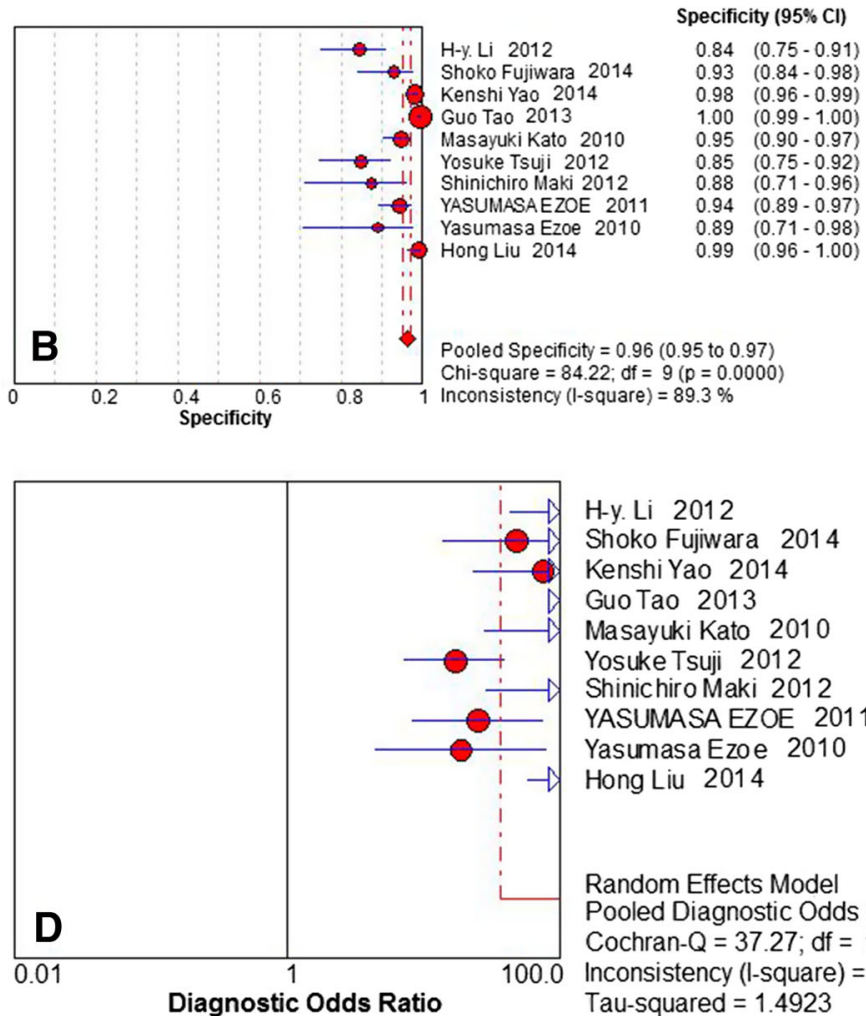

H-y. Li 2012

Shoko Fujwara 2014

Kenshi Yao 2014

Guo Tao 2013

Masayuki Kato 2010

Yosuke Tsuji 2012

Shinichiro Maki 2012

YASUMASA EZOE 2011

Yasumasa Ezoe 2010

Hong Liu 2014

Random Effects Model

Pooled Diagnostic Odds Ratio $=88.83$ (36.53 to 215.99)

Cochran- $Q=37.27 ; \mathrm{df}=9(p=0.0000)$

Inconsistency (l-square) $=75.8 \%$

Tau-squared $=1.4923$
Fig. 2 Per-lesion analysis of diagnostic performance of magnifying endoscopy with narrow band imaging (ME-NBI) for early gastric cancer (GC). a Pooled sensitivity for ME-NBI to differentiate GC. b Pooled specificity for ME-NBI to differentiate GC. c The summary

electronic supplementary material). The final pooled sensitivity was $0.82\left(95 \%\right.$ CI $\left.0.75-0.88 ; I^{2}=83.1 \%\right)$, the pooled specificity was $0.91 \quad(95 \%$ CI $0.87-0.94$; $\left.I^{2}=45.7 \%\right)$, the DOR was 39.52 (95\% CI 14.48-107.85; $I^{2}=57.1 \%$ ), the AUC was 0.93 (standard error 0.03 ), and $Q^{*}$ was 0.88 (standard error 0.03 ).

\section{The value of WLI in the diagnosis of early gastric cancer}

Four studies [16-19] evaluated the diagnostic efficacy of WLI in early gastric cancer. These studies included a total of 433 patients and 527 lesions. As shown in Fig. 3, the receiver operating characteristic (SROC) curve for diagnosis by MENBI. d Diagnostic odds ratio $(O R)$ for ME-NBI to differentiate GC. $A U C$ area under the curve, $C I$ confidence interval, $d f$ degrees of freedom, $S E$ standard error

pooled sensitivity of WLI in the diagnosis of gastric cancer was 0.48 (95\% CI $0.39-0.57 ; I^{2}=79.6 \%$ ), the pooled diagnostic specificity was $0.67 \quad(95 \%$ CI $0.62-0.71$; $\left.I^{2}=81.9 \%\right)$, the DOR was 2.10 (95\% CI $0.53-8.39$; $\left.I^{2}=82.4 \%\right)$, the AUC was 0.62 (standard error 0.11$)$, and $Q^{*}$ was 0.59 (standard error 0.08 ).

\section{Sensitivity analysis and meta-regression analysis}

As shown in Figs. 2 and 3, there was large heterogeneity of the pooled effect size from the original studies. Thus, sensitivity analysis on the efficacy of ME-NBI in the diagnosis of gastric cancer was performed according to 


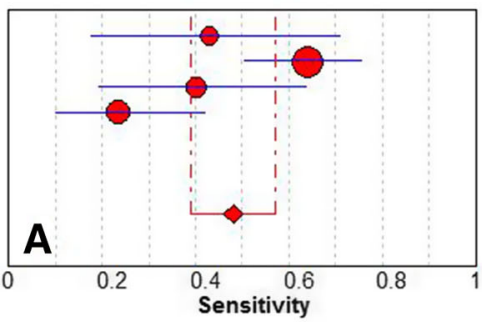

Masayuki Kato 2010 Shinichiro Maki 2012

YASUMASAEZOE 2011

Yasumasa Ezoe 2010

Pooled Sensitivity $=0.48(0.39$ to 0.57$)$ Chi-square $=14.69 ; \mathrm{df}=3(\mathrm{p}=0.0021)$ Inconsistency (l-square) $=79.6 \%$

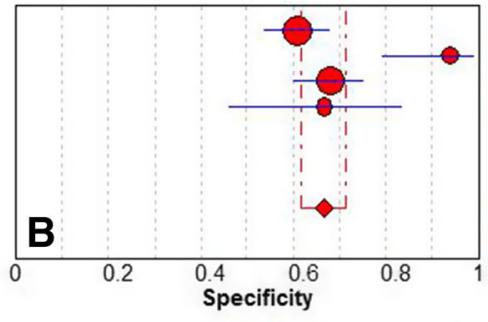

Masayuki Kato 2010 Shinichiro Maki 2012 YASUMASAEZOE 2011 Yasumasa Ezoe 2010

Pooled Specificity $=0.67(0.62$ to 0.71$)$ Chi-square $=16.54 ; \mathrm{df}=3(\mathrm{p}=0.0009)$ Inconsistency $(\mathrm{l}$-square $)=81.9 \%$

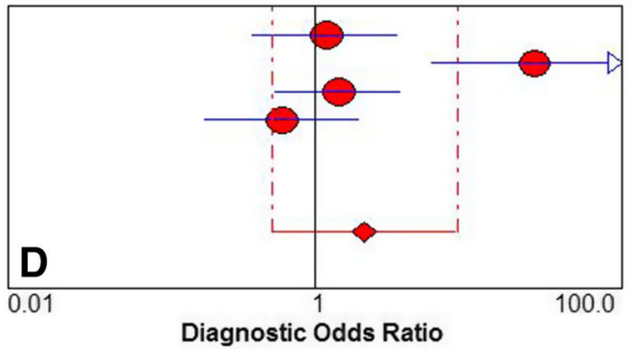

Masayuki Kato 2010 YASUMASAEZOE 2011 Yasumasa Ezoe 2010

Random Effects Model Tau-squared $=1.6305$
Sensitivity $(95 \% \mathrm{CI})$

$0.43(0.18-0.71)$

$0.64 \quad(0.51-0.76)$

$0.40 \quad(0.19-0.64)$

$0.23(0.10-0.42)$ Shinichiro Maki 2012

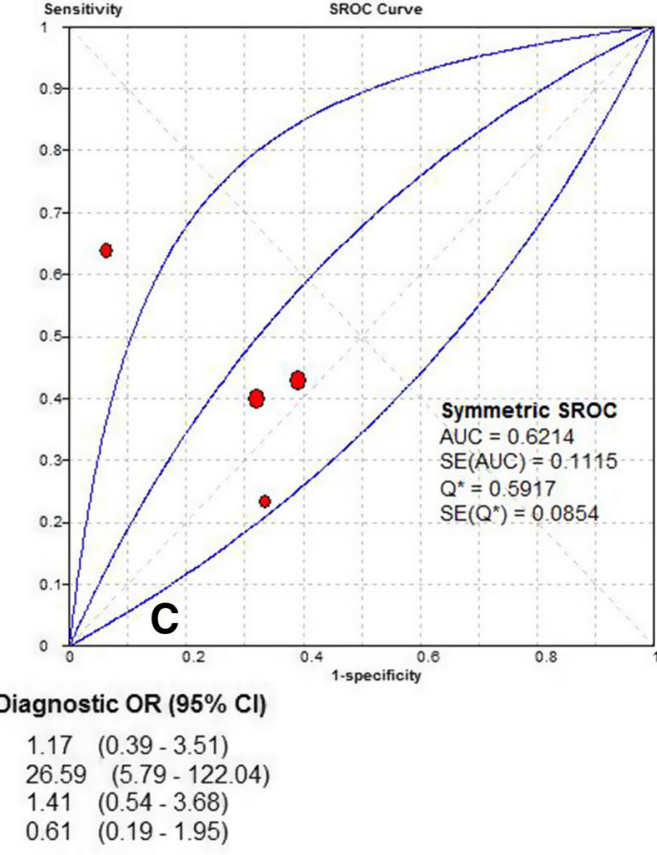

Pooled Diagnostic Odds Ratio $=2.10(0.53$ to 8.39$)$ Cochran-Q $=17.07 ; \mathrm{df}=3(\mathrm{p}=0.0007)$ Inconsistency (I-square) $=82.4 \%$
Fig. 3 Analysis of diagnostic performance of white light imaging (WLI) for gastric cancer (GC). a Pooled sensitivity for WLI to differentiate GC. b Pooled specificity for WLI to differentiate GC. c The summary receiver operating characteristic (SROC) curve for

the inclusion criterias of different studies (Table 3). The inclusion criterias were as follows: studies from Japan, single-center studies, prospective studies, a total sample size of more than 200,30 or more gastric lesions, lesion size of $10 \mathrm{~mm}$ of less, "VS classification" as the endoscopic diagnostic criteria, and literature quality score of 12 points or more. The results showed that the diagnostic efficacy of ME-NBI in gastric cancer was still high, and the stability of the results was very good. However, there was still significant heterogeneity among the studies. In addition, we sequentially excluded the following studies, which significantly deviated from the pooled effect size, to perform the analysis: the studies of Li et al. [13], Maki et al. [17], Tao et al. [21], and Kato et al. [16]. Sensitivity analyses were performed on the remaining eight, seven, and six studies. The results showed that the heterogeneity with respect to sensitivity was decreased significantly, whereas the heterogeneity with respect to specificity was not reduced significantly. However, the results still showed a high diagnostic efficacy of MENBI in gastric cancer, and the AUC ranged between 0.93 and 0.98 . diagnosis by WLI. d Diagnostic odds ratio (OR) for WLI to differentiate GC. AUC area under the curve, $C I$ confidence interval, $d f$ degrees of freedom, $S E$ standard error

The study country (from Japan, and not from Japan), publication year (before 2012, and after 2012), singlecenter/multicenter study, a total sample size of more than 200, "VS classification" as the endoscopic diagnostic criteria, and literature quality score of 12 points or more were selected for the meta-regression analysis. All these factors could be extracted from the ten studies. The study country and sample size were found to affect the heterogeneity. However, if studies from different countries and different sample sizes were selected for meta-analysis, the heterogeneity remained high. Meta-regression analysis did not solve the problem of heterogeneity (see "supplemental material 3" in the electronic supplementary material.).

\section{Assessment of publication bias}

To assess publication bias, we used weighted linear regression as provided by the statistical package Stata to determine funnel plot symmetry. The results showed that the slope (bias) was 2.732 , with $P=0.921$, indicating no statistical significance and no publication bias in the studies included. As shown in the Deeks funnel plot (Fig. 4), the 
Table 3 Outcomes of sensitivity analysis of selected studies for assessing the stability of diagnostic accuracy of magnifying endoscopy with narrow band imaging (ME-NBI) for early gastric cancer

\begin{tabular}{|c|c|c|c|c|c|c|c|}
\hline \multirow[t]{2}{*}{ Study characteristics } & \multirow{2}{*}{$\begin{array}{l}\text { No. of } \\
\text { studies }\end{array}$} & \multirow{2}{*}{$\begin{array}{l}\text { No. of } \\
\text { patients }\end{array}$} & \multicolumn{2}{|c|}{ Pooled estimates $^{c}$} & \multicolumn{2}{|c|}{ Inconsistency } & \multirow[t]{2}{*}{ AUC } \\
\hline & & & Sensitivity & Specificity & $\begin{array}{l}I^{2} \text { for } \\
\text { sensitivity } \\
(\%)\end{array}$ & $\begin{array}{l}I^{2} \text { for } \\
\text { specificity } \\
(\%)\end{array}$ & \\
\hline Study from Japan $[14,16-20,23]$ & 7 & 980 & $\begin{array}{l}0.78 \\
\quad(0.73-0.83)\end{array}$ & $\begin{array}{l}0.95 \\
\quad(0.93-0.96)\end{array}$ & 75.1 & 74.6 & 0.93 \\
\hline Single-center study $[13,16,17,19-23]$ & 8 & 1237 & $\begin{array}{l}0.86 \\
\quad(0.82-0.90)\end{array}$ & $\begin{array}{l}0.96 \\
\quad(0.95-0.97)\end{array}$ & 76.9 & 91.1 & 0.97 \\
\hline Prospective study $[13,14,16,18,19,22]$ & 6 & 887 & $\begin{array}{l}0.82 \\
\quad(0.76-0.87)\end{array}$ & $\begin{array}{l}0.96 \\
\quad(0.94-0.97)\end{array}$ & 84.2 & 84.8 & 0.96 \\
\hline Sample size $>200[14,16,21,22]$ & 4 & 1019 & $\begin{array}{l}0.81 \\
\quad(0.70-0.89)\end{array}$ & $\begin{array}{l}0.98 \\
\quad(0.98-0.99)\end{array}$ & 64.5 & 83.2 & 0.98 \\
\hline $\begin{array}{l}\text { Number of cancerous lesions } \geq 30[13,17,19,20 \text {, } \\
23]\end{array}$ & 5 & 528 & $\begin{array}{l}0.86 \\
\quad(0.81-0.90)\end{array}$ & $\begin{array}{l}0.87 \\
\quad(0.83-0.91)\end{array}$ & 86.0 & 0.0 & 0.94 \\
\hline Lesion size $\leq 10 \mathrm{~mm}[14,16,18-21]$ & 6 & 1258 & $\begin{array}{l}0.75 \\
\quad(0.67-0.82)\end{array}$ & $\begin{array}{l}0.97 \\
\quad(0.96-0.98)\end{array}$ & 59.6 & 85.3 & 0.96 \\
\hline VS standard $[14,16-21,23]^{\mathrm{a}}$ & 8 & 1488 & $\begin{array}{l}0.80 \\
\quad(0.74-0.84)\end{array}$ & $\begin{array}{l}0.97 \\
\quad(0.96-0.97)\end{array}$ & 74.0 & 88.0 & 0.95 \\
\hline Study quality score $\geq 12[13,14,18,21-23]$ & 6 & 1368 & $\begin{array}{l}0.82 \\
\quad(0.76-0.87)\end{array}$ & $\begin{array}{l}0.97 \\
\quad(0.96-0.98)\end{array}$ & 84.1 & 93.1 & 0.97 \\
\hline Eight studies $[14,16,18-23]^{\mathrm{b}}$ & 8 & 1485 & $\begin{array}{l}0.75 \\
\quad(0.69-0.81)\end{array}$ & $\begin{array}{l}0.97 \\
\quad(0.96-0.98)\end{array}$ & 44.3 & 87.7 & 0.96 \\
\hline Seven studies $[14,16,18-20,22,23]^{\mathrm{b}}$ & 7 & 977 & $\begin{array}{l}0.73 \\
\quad(0.67-0.79)\end{array}$ & $\begin{array}{l}0.96 \\
\quad(0.94-0.97)\end{array}$ & 23.6 & 79.9 & 0.94 \\
\hline Six studies $[14,18-20,22,23]^{\mathrm{b}}$ & 6 & 866 & $\begin{array}{l}0.72 \\
\quad(0.65-0.78)\end{array}$ & $\begin{array}{l}0.96 \\
(0.94-0.97)\end{array}$ & 0.0 & 83 & 0.93 \\
\hline
\end{tabular}

${ }^{a}$ VS classification criteria used as the criteria for ME-NBI diagnosis of early gastric cancer

b Some studies, significantly deviating from the pooled effect size, were excluded, and the sensitivity analyses were performed on the remaining eight, seven, and six studies.

c The $95 \%$ confidence interval is given in parentheses.

Fig. 4 Deeks funnel plot to evaluate publication bias

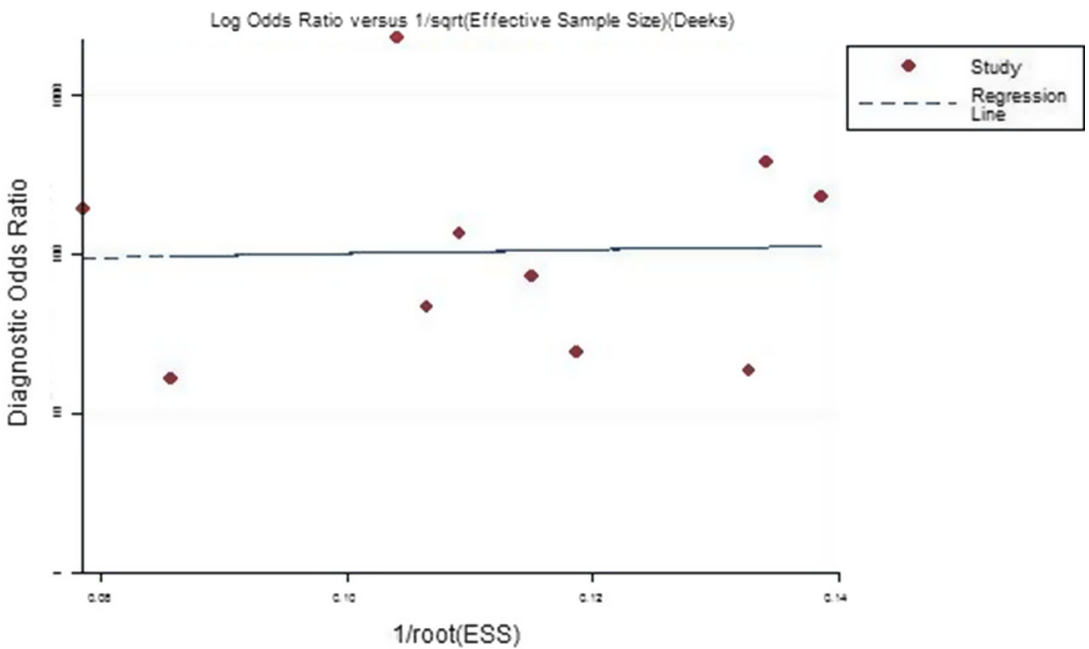


angle of the regression line and the DOR axis was nearly $90^{\circ}$, which indicated the absence of publication bias among the studies included.

\section{Discussion}

The results of this study show that although there are certain differences regarding the sensitivity of ME-NBI for the diagnosis of early gastric cancer, its diagnostic efficacy is very high. In contrast, WLI has poor performance, low sensitivity, and low specificity.

By means of ME-NBI examination, one is immediately able to observe suspicious lesions in higher magnification, and can clearly see the micromorphology and the microvascular structure of mucosal lesions to identify cancerous lesions [24, 25]. Currently, the "VS classification" criteria of Yao et al. [26, 27], which suggest the presence of a lesion with the boundary, an abnormal vascular network on its surface, or an abnormal change on the surface of the microstructure, are widely used as the diagnostic criteria for ME-NBI diagnosis of gastric cancer. On the basis of this standard, published studies have reported that ME-NBI has a high diagnostic efficacy. However, there is a large difference in the sensitivity among these studies, ranging from 0.60 to 0.97 [13, 14, 18]. To our knowledge, our study is the first meta-analysis focusing on systematically evaluating the diagnostic efficacy of MENBI for early gastric cancer. In the ten studies included in the meta-analysis, six investigated lesions with mean size of $10 \mathrm{~mm}$ or less. Endoscopic biopsies may be difficult to perform for some smaller gastric lesions, especially micro gastric cancer, and a false negative result may occur. There seem have been no studies focusing on the accuracy of small gastric cancer biopsy. Some reports have demonstrated there are certain differences between the pathologic findings for biopsy specimens and ESD specimens, with a reported incompatible rate of 27.1-44.5\% [28]. ME-NBI can assist in targeting the biopsy site, and immediate endoscopic diagnosis of gastric cancer by MENBI can be considered as an optical biopsy [14, 27, 29]. However, the performance of ME-NBI is also affected by the blood and bile in the stomach. Since the working principle of narrow band imaging is based on the strong absorption of narrow band light by hemoglobin and the high reflection of mucosal surfaces, blood and bile on the surface of lesions may result in failure of ME-NBI observation $[24,30]$.

Use of ME-NBI is simple for general endoscopists to perform, is a mature endoscopic diagnostic method, and the diagnostic standard for ME-NBI in diagnosing early gastric cancer has been defined-the relatively widely used diagnostic standard being the "VS classification" criteria proposed by Yao et al. In addition, we believe that the endoscopic characteristics of both early gastric cancer and late-stage gastric cancer under ME-NBI should comply with the "VS classification" criteria. ME-NBI has a high diagnostic performance in distinguishing cancer from benign lesions. However, there are limitations in assessment of the depth of invasion. Some patients still need further surgery after gastric ESD or endoscopic mucosal resection [13].

Clinical studies regarding the application of WLI in the diagnosis of gastric cancer are relatively rare, and only four studies were included in the current meta-analysis [16-19]. The results of this study showed that WLI has lower sensitivity and specificity than ME-NBI. Typically, lesions were first observed by WLI, and then further examined by using magnifying endoscopy to further clarify their characteristics. Therefore, careful observation is also important for the application of WLI. In addition, the morphological characteristics of lesions under WLI are also helpful for the diagnosis of gastric cancer [16, 18]. An irregular margin and a spiny depressed area in a lesion under WLI indicate the possibility of malignant change. WLI combined with ME-NBI exhibits high sensitivity (up to 0.95 ) in the diagnosis of gastric cancer [18].

There are some limitations of the current study. There was large heterogeneity among the studies included. The source of the heterogeneity was explored by meta-regression analysis, but meta-regression analysis did not solve the problem of heterogeneity (see "supplemental material 3" in the electronic supplementary material). The poor performance of meta-regression analysis might be because the sample size of the studies included was too small and possible other factors affecting the heterogeneity were not analyzed. The factors selected for the meta-regression analysis should be extracted from all the studies, but some factors, only referred to in some of the studies, could not be analyzed. In the ten studies included, although they all studied gastric cancer, the characteristics of gastric cancer differed among the studies. For example, some studies targeted adenocarcinoma and some studies targeted small gastric cancer. Each study targeted patients with gastric cancer or gastric illnesses, rather than the general population, which inevitably led to the differences of sensitivity, ranging from 0.60 to 0.97 . This may also be an important cause of the heterogeneity. In addition, it requires considerable experience to use ME-NBI for diagnosis of gastric cancer. Studies have shown that advanced training can improve the diagnostic rate of early gastric cancer [31,32]. Therefore, the differences in the experience level of the endoscopists among the studies included may also be a factor contributing to heterogeneity. Only four studies [13, $14,16,20]$ clearly described the experience level of the endoscopists, and there were significant differences 
regarding the level of experience with respect to the total number of cases and the time spent performing ME-NBI among these studies. Moreover, there were significant differences in the experience level of the endoscopists in a single study. For example, the kappa values of endoscopists for the diagnosis of gastric cancer in the studies by Li et al. [13], Fujiwara et al. [20], and Liu et al. [22] were 0.883, 0.56 , and 0.715 , respectively. In addition, of the ten studies meeting the inclusion criteria, seven are from Japan, which may to some degree be related to the heterogeneity among the studies. This study also analyzed the diagnostic efficacy of WLI for gastric cancer. However, clinical studies on WLI in gastric cancer diagnosis were relatively rare, and there was large heterogeneity among the studies.

A sensitivity analysis was also performed to solve the problem of heterogeneity, and to evaluate the stability of the conclusions. Whereas the sensitivity analysis found the heterogeneity in sensitivity could be significantly reduced, the heterogeneity with respect to specificity did not change significantly. Nevertheless, the results of the sensitivity analysis were solid, ranging from 0.93 to 0.98 . These data indicate the results of this meta-analysis are reliable.

\section{Conclusion}

In summary, WLI has poor performance in the diagnosis of early gastric cancer. ME-NBI is an effective tool for realtime endoscopic diagnosis of early gastric cancer. Since there was large heterogeneity among the studies included, the diagnostic efficacy of ME-NBI for early gastric cancer may need to be investigated further.

\section{References}

1. Jemal A, Bray F, Center MM, Ferlay J, Ward E, Forman D. Global cancer statistics. CA Cancer J Clin. 2011;61:69-90.

2. Ferlay J, Soerjomataram I, Dikshit R, et al. Cancer incidence and mortality worldwide: sources, methods and major patterns in GLOBOCAN 2012. Int J Cancer. 2015;136:359-86.

3. Katai H, Sano T. Early gastric cancer: concepts, diagnosis, and management. Int J Clin Oncol. 2005;10:375-83.

4. Crew KD, Neugut AI. Epidemiology of gastric cancer. World J Gastroenterol. 2006;12:354-62.

5. Tsubono Y, Hisamichi S. Screening for gastric cancer in Japan. Gastric Cancer. 2000;3:9-18.

6. Yeoh KG. How do we improve outcomes for gastric cancer? J Gastroenterol Hepatol. 2007;22:970-2.

7. Isomoto H, Shikuwa S, Yamaguchi N, Fukuda E, Ikeda K, Nishiyama H, et al. Endoscopic submucosal dissection for early gastric cancer: a large-scale feasibility study. Gut. 2009;58:331-6.

8. Goto O, Fujishiro M, Kodashima S, Ono S, Omata M. Outcomes of endoscopic submucosal dissection for early gastric cancer with special reference to validation for curability criteria. Endoscopy. 2009;41:118-22.

9. Lee H, Yun WK, Min BH, Lee JH, Rhee PL, Kim KM, et al. A feasibility study on the expanded indication for endoscopic submucosal dissection of early gastric cancer. Surg Endosc. 2011;25:1985-93.

10. Cunningham SC, Kamangar F, Kim MP, Hammoud S, Haque R, Maitra A, et al. Survival after gastric adenocarcinoma resection: eighteen-year experience at a single institution. J Gastrointest Surg. 2005;9:718-25.

11. Fayçal J, Bessaguet C, Nousbaum JB, Cauvin JM, Cholet F, Bideau K, et al. Epidemiology and long term survival of gastric carcinoma in the French district of Finistere between 1984 and 1995. Gastroenterol Clin Biol. 2005;29:23-32.

12. Hamashima C, Okamoto M, Shabana M, Osaki Y, Kishimoto T. Sensitivity of endoscopic screening for gastric cancer by the incidence method. Int J Cancer. 2013;133:653-9.

13. Li HY, Dai J, Xue HB, Zhao YJ, Chen XY, Gao YJ, et al. Application of magnifying endoscopy with narrow-band imaging in diagnosing gastric lesions: a prospective study. Gastrointest Endosc. 2012;76:1124-32.

14. Yao K, Doyama H, Gotoda T, Ishikawa H, Nagahama T, Yokoi $\mathrm{C}$, et al. Diagnostic performance and limitations of magnifying narrow-band imaging in screening endoscopy of early gastric cancer: a prospective multicenter feasibility study. Gastric Cancer. 2014;17:669-79.

15. Whiting P, Rutjes AW, Reitsma JB, Bossuyt PM, Kleijnen J. The development of QUADAS: a tool for the quality assessment of studies of diagnostic accuracy included in systematic reviews. BMC Med Res Methodol. 2003;3:25.

16. Kato M, Kaise M, Yonezawa J, Toyoizumi H, Yoshimura N, Yoshida Y, et al. Magnifying endoscopy with narrow-band imaging achieves superior accuracy in the differential diagnosis of superficial gastric lesions identified with white-light endoscopy: a prospective study. Gastrointest Endosc. 2010;72:523-9.

17. Maki S, Yao K, Nagahama T, Beppu T, Hisabe T, Takaki Y, et al. Magnifying endoscopy with narrow-band imaging is useful in the differential diagnosis between low-grade adenoma and early cancer of superficial elevated gastric lesions. Gastric Cancer. 2013;16:140-6.

18. Ezoe Y, Muto M, Uedo N, Doyama H, Yao K, Oda I, et al. Magnifying narrowband imaging is more accurate than conventional white-light imaging in diagnosis of gastric mucosal cancer. Gastroenterology. 2011;141:2017-25.

19. Ezoe Y, Muto M, Horimatsu T, Minashi K, Yano T, Sano Y, et al. Magnifying narrow-band imaging versus magnifying white-light imaging for the differential diagnosis of gastric small depressive lesions: a prospective study. Gastrointest Endosc. 2010;71:477-84.

20. Fujiwara S, Yao K, Nagahama T, Uchita K, Kanemitsu T, Tsurumi $\mathrm{K}$, et al. Can we accurately diagnose minute gastric cancers ( $\leq 5 \mathrm{~mm})$ ? Chromoendoscopy (CE) vs magnifying endoscopy with narrow band imaging (M-NBI). Gastric Cancer. 2014. doi:10.1007/s10120-014-0399-2.

21. Tao G, Xing-Hua L, Ai-Ming Y, Wei-Xun Z, Fang Y, Xi W, et al. Enhanced magnifying endoscopy for differential diagnosis of superficial gastric lesions identified with white-light endoscopy. Gastric Cancer. 2014;17:122-9.

22. Liu H, Wu J, Lin XC, Wei N, Lin W, Chang H, et al. Evaluating the diagnoses of gastric antral lesions using magnifying endoscopy with narrow-band imaging in a Chinese population. Dig Dis Sci. 2014;59:1513-9.

23. Tsuji Y, Ohata K, Sekiguchi M, Ohno A, Ito T, Chiba H, et al. Magnifying endoscopy with narrow-band imaging helps 
determine the management of gastric adenomas. Gastric Cancer. 2012;15:414-8.

24. Gono K, Obi T, Yamaguchi M, Ohyama N, Machida H, Sano Y, et al. Appearance of enhanced tissue features in narrow-band endoscopic imaging. J Biomed Opt. 2004;9:568-77.

25. Nakayoshi T, Tajiri H, Matsuda K, Kaise M, Ikegami M, Sasaki H. Magnifying endoscopy combined with narrow band imaging system for early gastric cancer: correlation of vascular pattern with histopathology. Endoscopy. 2004;36:1080-4.

26. Yao K, Takaki Y, Matsui T, Iwashita A, Anagnostopoulos GK, Kaye $\mathrm{P}$, et al. Clinical application of magnification endoscopy and narrow-band imaging in the upper gastrointestinal tract: new imaging techniques for detecting and characterizing gastrointestinal neoplasia. Gastrointest Endosc Clin N Am. 2008;18:415-33.

27. Yao K, Anagnostopoulos GK, Ragunath K. Magnifying endoscopy for diagnosing and delineating early gastric cancer. Endoscopy. 2009;41:462-7.

28. Lim H, Jung HY, Park YS, Na HK, Ahn JY, Choi JY, et al. Discrepancy between endoscopic forceps biopsy and endoscopic resection in gastric epithelial neoplasia. Surg Endosc. 2014;28:1256-62.

29. Jiang H, Tu HM, Qiao Q, Xu KB, Li J, Qi XW, et al. Effect of route of preoperative biopsy on endoscopic submucosal dissection for patients with early gastric cancer. Asian Pac J Cancer Prev. 2014;15:8917-21.

30. Kuznetsov K, Lambert R, Rey JF. Narrow-band imaging: potential and limitations. Endoscopy. 2006;38:76-81.

31. Dias-Silva D, Pimentel-Nunes P, Magalhães J, Magalhães R, Veloso N, Ferreira C, et al. The learning curve for narrow-band imaging in the diagnosis of precancerous gastric lesions by using Web-based video. Gastrointest Endosc. 2014;79:910-20; quiz 983.e1, 983.e4..

32. Higashi R, Uraoka T, Kato J, Kuwaki K, Ishikawa S, Saito Y, et al. Diagnostic accuracy of narrow-band imaging and pit pattern analysis significantly improved for less-experienced endoscopists after an expanded training program. Gastrointest Endosc. 2010;72:127-35. 